\title{
Hydrogen-Bond Dynamics and Energetics of Biological Water
}

Aniruddha Adhikari, ${ }^{[a, b]}$ Won-Woo Park, ${ }^{[a]}$ and Oh-Hoon Kwon ${ }^{*[a, c]}$

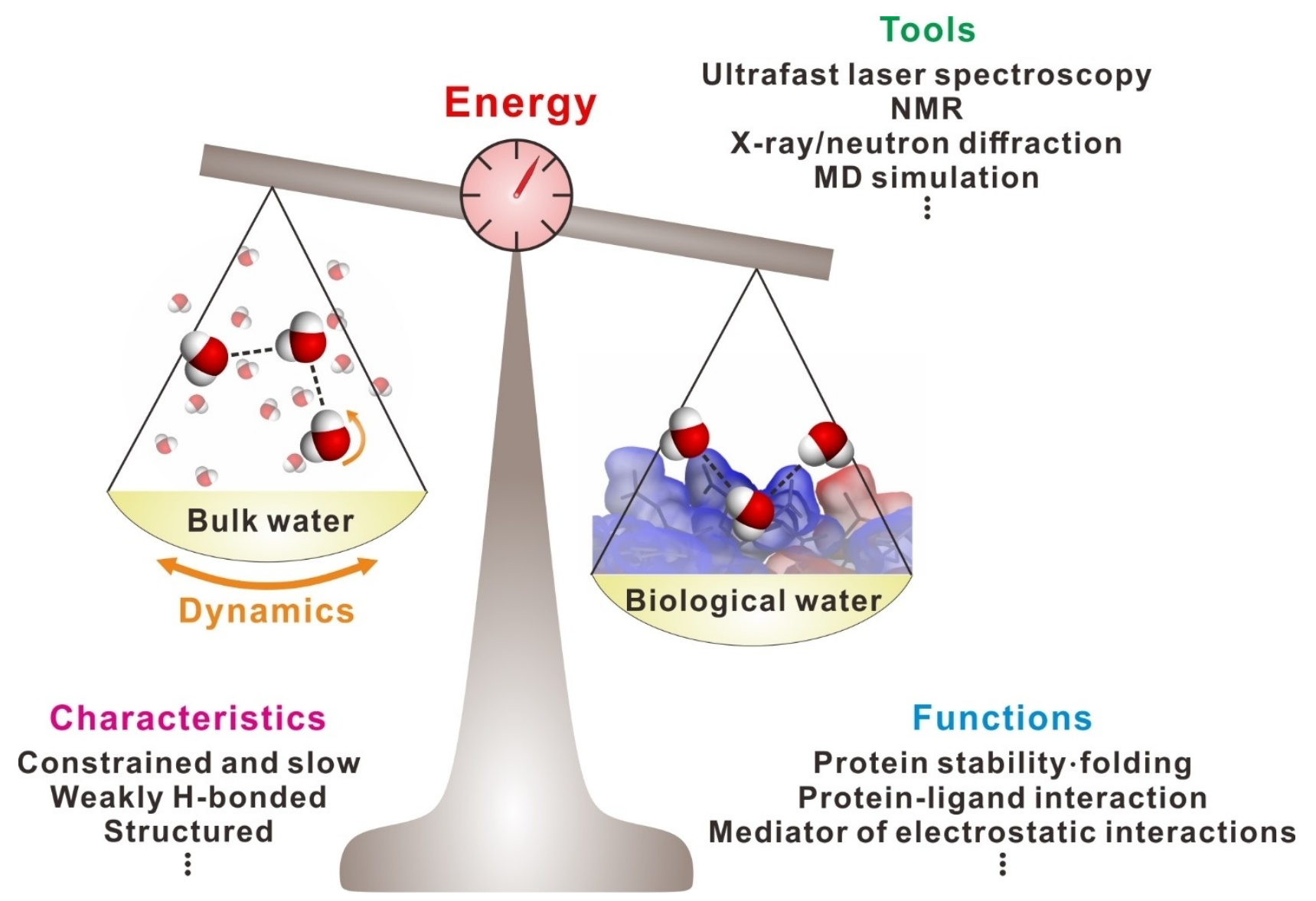


Water molecules in the immediate vicinity of biomacromolecules and biomimetic organized assemblies often exhibit a markedly distinct behavior from that of their bulk counterparts. The overall sluggish behavior of biological water substantially affects the stability and integrity of biomolecules, as well as the successful execution of various crucial water-mediated biochemical phenomena. In this Minireview, insights are provided into the features of truncated hydrogen-bond networks that grant biological water its unique characteristics. In particular, experimental results and theoretical investigations, based on chemical kinetics, are presented that have shed light on the dynamics and energetics governing such characteristics. It is emphasized how such details help us to understand the energetics of biological water, an aspect relatively less explored than its dynamics. For instance, when biological water at hydrophilic or charged protein surfaces was explored, the free energy of $\mathrm{H}$-bond breakage was found to be of the order of $0.4 \mathrm{kcal} \mathrm{mol}^{-1}$ higher than that of bulk water.

\section{Biological Water}

\subsection{Definition}

The role of water as a vital contributor in biological processes is a widely acknowledged fact. Numerous water-mediated phenomena prevalent in biotic environments demonstrate that water often promotes the fruitful execution of physiochemical processes between participant species, instead of serving as a passive solvent medium ${ }^{[1-9]}$. Unsurprisingly, some commentators have bestowed it with the status of a biomolecule. Water molecules present in the vicinity of biological systems are often characterized by unique interactions that grant them significantly different properties from those of bulk water. Despite the diverse profiles of biological entities, ranging from the surfaces of lipid bilayers, ${ }^{[10]}$ channel proteins, ${ }^{[1]]}$ and grooves of DNA ${ }^{[12]}$ to deeply buried active sites within enzymes, ${ }^{[13,14]}$ there are certain common features and unique characteristics that distinguish water molecules in such environments. These characteristics typically stem from the fact that the extended hydrogen $(\mathrm{H})$ bond network in bulk water is severely curtailed at biological interfaces, and it is often insightful to treat the relevant water molecules as a distinct category under the term "biological water". ${ }^{[15-33]}$

However, the use of the term biological water is often contested. The point of contention appears to be whether or not water near bio-macromolecules can be accorded special status as a distinct entity imparting and regulating a range of properties necessary for effective functioning of biomolecules. Alternatively, it is possible that it should simply be regarded as water, with its distinctness originating from the properties of neighboring macromolecules. Therefore, the tendency to overemphasize the uniqueness of biological water, rather than consider the effects of the biomolecules, should be avoided. ${ }^{[5]}$

[a] Prof. Dr. A. Adhikari, W.-W. Park, Prof. Dr. O.-H. Kwon Department of Chemistry

Ulsan National Institute of Science and Technology (UNIST) 44919 Ulsan (Republic of Korea)

E-mail: ohkwon@unist.ac.kr

[b] Prof. Dr. A. Adhikari

Department of Physics

UNIST

44919 Ulsan (Republic of Korea)

[c] Prof. Dr. O.-H. Kwon

Center for Soft and Living Matter

Institute for Basic Science (IBS)

44919 Ulsan (Republic of Korea)

\subsection{Features}

Although biological water is often understood as biomoleculeassociated water, its features can also appear in confined media that mimic these environments. Thus, behavior akin to biological water can be found in a wide variety of abiotic organized assemblies such as micelles, microemulsions, cyclodextrins, surfactant polymer aggregates, zeolites, and gels. In fact, the aforenoted assemblies are often used as simple model systems to study and elucidate the role of such water molecules in the functioning of various bio-macromolecules. ${ }^{[16,19]}$

The following are some of the general attributes of biological water. ${ }^{[1]}$ First, biological water occurs in constrained geometries that forbid three-dimensional, and at times, even two-dimensional, extended $\mathrm{H}$-bond networks. It often lacks adequate water molecules in its neighborhood and exhibits diminished coordination. Second, it may form local structures that represent configurations with local free-energy minima. Third, depending on the polarity of the bio-surface it inhabits, the strength of the $\mathrm{H}$-bonding of biological water may vary; in this regard, the two extreme cases correspond to relatively immobilized (or "bound") and relatively "free" biological water. Both types of biological water molecules are possibly subject to dynamic exchange between their sub-populations. ${ }^{[15,16]}$ Fourth, because of such variations in $\mathrm{H}$-bonding strengths, the dynamics of biological water may entail a substantially wider time range than that of bulk water, which is often characterized by relaxation functions that are non-exponential. ${ }^{[29]}$

The subtle interplay among the various characteristics of biological water results in highly heterogeneous hydration environments with short lengths and timescales, thereby yielding interesting consequences. ${ }^{[30]}$ For instance, the clustering of biological water molecules near a protein surface can enhance the local density of the water molecules by almost 1.25 times that of bulk water. ${ }^{[34]}$ The disrupted water-water network can prevent the freezing of such protein-bound water at temperatures well below the freezing point of bulk water, with important implications for certain life forms. Further, the lack of mutual polarization among $\mathrm{H}$-bonded water molecules leads to a loss of the effective dipole moment and results in a lower dielectric constant, making biological water substantially less polar than bulk water; this in turn affects the solvation behavior of ions in its neighborhood. ${ }^{[23]}$

One of the most distinguishing features of biological water is the sluggishness of its dynamics with respect to that of bulk water. This sluggishness is sometimes attributed to the 
maintenance of dynamic equilibrium between the "bound" and "free" water molecules, which is a process that entails overcoming an activation energy barrier (Figure 1a); consequently, this process incurs energetic costs in terms of the breaking and reforming of relevant $\mathrm{H}$-bonds. ${ }^{[15]}$ The strengths of the $\mathrm{H}$-bonds involved in these restructuring events are chiefly influenced by the polarity of the participating moieties such as the residues and side chains of amino acids constituting the proteins. Other factors such as ambient temperature, pressure, $\mathrm{pH}$, or presence of additives also dictate the kinetics of such exchanges and influence the sluggishness of the dynamics. The characteristic sluggishness of biological water has also been explained in terms of the mobility of hydration layer water molecules that are strongly coupled to the local protein domain motions (Figure 1b). ${ }^{[35]}$ The ultrafast dynamics on early timescales characterizes local collective water-network relaxation. On longer timescales, the new $\mathrm{H}$-bond networks are established, and this is accompanied by local protein fluctuations and bulkwater dynamic exchange. Both processes are correlated with the local chemical identity and structural flexibility of the protein.

\subsection{Functions}

In the case of proteins, the preferential solvation of polar moieties along the peptide backbone owing to bound water helps maintain the integrity of protein structures as well as their functions that arise through reduced competitive solvation from neighboring ions and counterions. However, such interactions also depend on the location of the biological water. In contrast with biological water existing on protein surfaces, water molecules buried deep within the cavities of the protein interior can form structured cages near non-polar moieties facilitating the entropic stabilization of the system. ${ }^{[36]}$

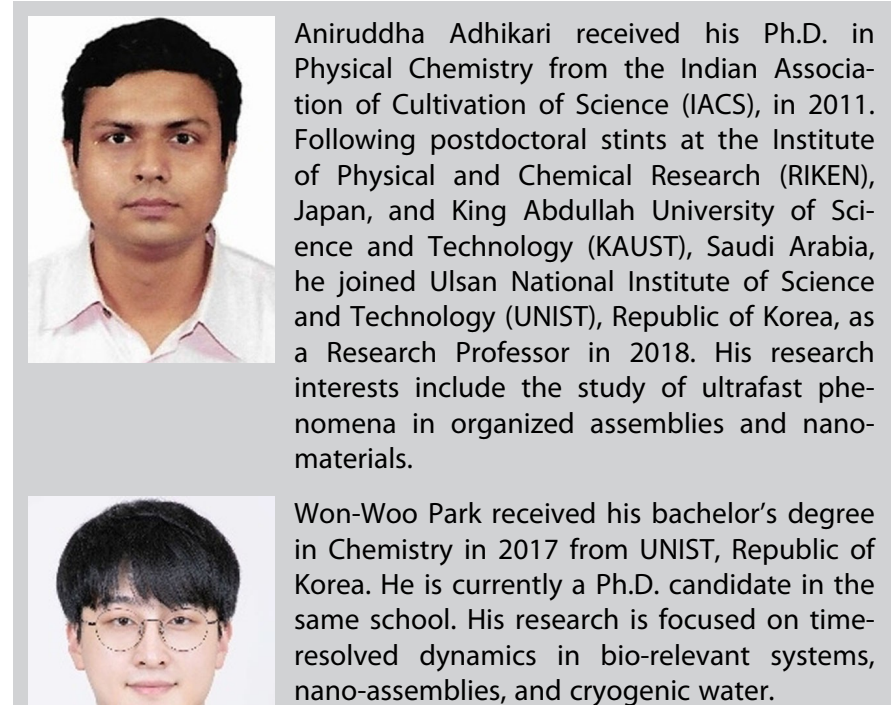

Aquaporins are a class of membrane proteins that serve as water channels. They are noted for their unique ability to maintain membrane potential and intracellular $\mathrm{pH}$ via the efficient and selective passage of water molecules and small solutes through their pores. ${ }^{[1]}$ Although proton transfer is expected to occur efficiently via water bridges through protein pores, aquaporins are impermeable to proton transport. This fascinating contradiction arises from the unique characteristics of the $\mathrm{H}$-bond network within the pore, where one of the water molecules residing toward the center of the water network is constrained by the electrostatic influence of the adjoining protein residues in such a fashion that it generates oppositely oriented neighboring water molecules in the two halves of the channel. This prevents the formation of a "proton wire," the pre-requisite for the Grotthuss conductance of protons, without affecting the ability of water molecules to diffuse through by themselves. ${ }^{[37]}$

Green fluorescent protein (GFP), a marker molecule employed extensively in bio-medical research, owes its characteristic emission to the excited-state proton transfer (ESPT) behavior of its intrinsic chromophore. ${ }^{[38]}$ The success of the ESPT process was found to be dependent on the intervening water molecule that forms the bridge connecting the labile proton to its destination; moreover, such a water molecule can be exchanged with bulk water residing beyond the confines of the $\beta$-barrel structure enclosing the chromophore. ${ }^{[39]}$ In contrast, in D-luciferin, the molecule responsible for bioluminescence in fireflies, ESPT to the surrounding water molecules can occur quite efficiently. ${ }^{[40]}$

Biological water in the vicinity of metalloenzyme active sites plays a crucial role in governing the kinetics of catalytic steps. For instance, single-point mutation in native human carbonic anhydrase II can lead to the restructuring of water replenishment pathways that influence the electrostatic environment around the relevant reaction centers. ${ }^{[13]}$ Such reorganization of

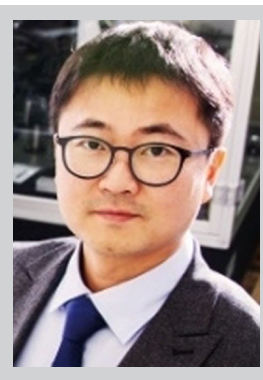

Oh-Hoon Kwon received his Ph.D. in Physical Chemistry from Seoul National University in 2004. After serving as a Postdoctoral Scholar and Senior Scientist at Caltech, USA, under Prof. Ahmed H. Zewail, he joined UNIST, Republic of Korea, as an Assistant Professor in 2013. He is currently an Associate Professor in the Department of Chemistry, leading both the femtosecond-resolved optical spectroscopy and electron microscopy labs. His research interests include the electronic and structural dynamics of elementary reactions in condensed phases with emphasis on nonequilibrium photo-induced phase transformations of materials, electron-phonon interactions in correlated materials, photo-induced dynamics in nanometer-sized materials, ultrafast chemical dynamics, and ultrafast hydration dynamics in biological systems. 
a)

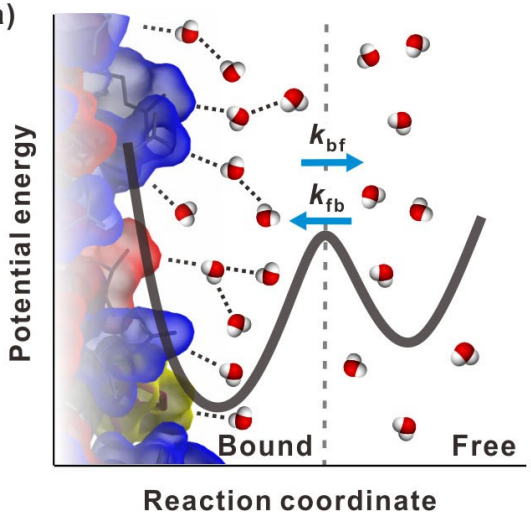

b)

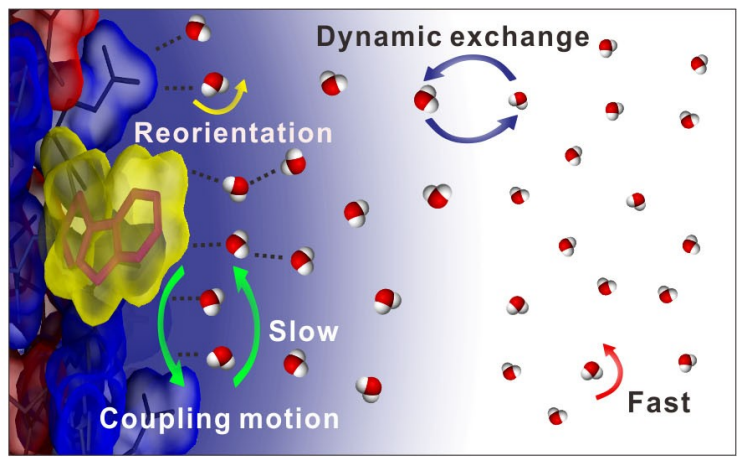

Figure 1. Schematic of the models used to investigate the slow relaxation of biological water. a) Dynamic exchange involving equilibrium between bound and free water molecules. Modified from Ref. [15], copyright 1997 and Ref. [33], copyright 2003, with permission from the American Chemical Society. b) Coupled protein-water fluctuation. Modified from Ref. [35], copyright 2009, with permission from the American Chemical Society.

the water network also occurs when the coordination geometries of the active-site metal ions are altered in response to the substitution of native metal ions by their non-native analogues. ${ }^{[14]}$

\section{Dynamics and Energetics}

\subsection{Methods}

Diffraction-based experiments helped establish the presence of biological water within protein structures; furthermore, dielectric relaxation measurements $s^{[41,42]}$ and nuclear magnetic resonance (NMR) spectroscopy ${ }^{[43]}$ provided the earliest evidence for the dynamic nature of biological water, highlighting its sluggishness in comparison to bulk water. Several time-resolved spectroscopic techniques such as UV/visible (Figure 2), infrared, and terahertz spectroscopies have also aided in gaining better
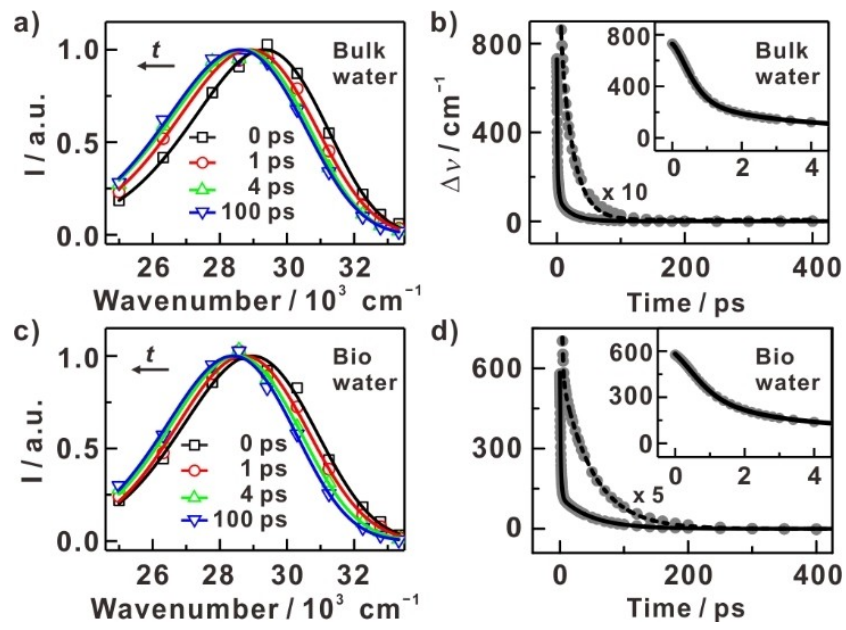

Figure 2. Time-resolved emission spectra and decay of solvent correlation function for water in bulk (top) and (bottom) vicinity of a model coiled-coil protein. Reproduced from Ref. [54], copyright 2020, with permission from Wiley-VCH Verlag GmbH \& Co. insights into the dynamics of biological water. ${ }^{[44-54]}$ These techniques, along with computational efforts, have been used to obtain theoretical underpinnings for many experimental observations. ${ }^{[35,44,46,55,56]}$ We now turn our attention to the hydration behavior of water near proteins to illustrate the intricacies involved in the $\mathrm{H}$-bond dynamics and energetics of biological water.

\subsection{Experimental Studies}

The dynamic-exchange model entailing the crossing of the activation energy barrier is one of the models used to explain the sluggishness of biological water. It is often insightful to obtain information regarding hydration at protein surfaces by probing biomimetic interfaces such as those present in micelles. Several ultrafast fluorescence-based time-resolved studies have been performed in this regard.

Sen et al. conducted a temperature-dependent study in a TX-100 micellar solution using a fluorophore (4-aminopthalimide, 4-AP) and observed the acceleration of solvation dynamics on increasing the temperature. ${ }^{[47]}$ They proposed that the solvation dynamics follows an Arrhenius-type temperature dependence and plotted the inverse of the slow component solvation against the inverse of the temperature to obtain an activation energy of $\sim 9 \mathrm{kcal} \mathrm{mol}^{-1}$. As the plot was linear, they concluded that the temperature dependence was largely unaffected by changes in the structure and hydration state of the micelles. A subsequent solvation dynamics study on a micellar medium, by Kumbhakar et al., found an anomalous temperature dependence that did not conform to the activation energy barrier crossing model. ${ }^{[48]}$ They attributed the observed discrepancies to the choice of fluorophore used in these studies. According to this hypothesis, 4-AP, owing to its hydrophilicity, does not penetrate the interior of the palisade layer of micelles; consequently, no significant temperature dependence of the hydration structure was observed. Using more hydrophobic probes such as coumarin (C-153), they observed that solvation dynamics shows a strong temperature 
dependence and reported an inversion of the trend at $298 \mathrm{~K}$. They proposed that the non-Arrhenius nature of the temperature dependence arose from the critical interplay of the micellar size and the corresponding hydration rates. A more holistic picture was provided by Mitra et al., who showed that such solvation dynamics studies should involve a wider temperature range and ensure the structural integrity of the host micelle without influencing the residence site of the dye being used to report on the hydration behavior. ${ }^{[45]}$ They observed an Arrhenius-type behavior of the temperature-dependent hydration dynamics and re-evaluated the activation energy barrier to be $3.5 \mathrm{kcal} \mathrm{mol}^{-1}$, in agreement with computational studies.

The macromolecular motion can also contribute to the sluggishness of water dynamics in constrained geometries. For instance, using the time-dependent Stokes shift of a fluorophore confined within the cavity of gamma-cyclodextrin $(\gamma-C D)$, a toroidal shaped molecule, Vajda et al. showed that the solvation response of the confined water molecules can range from femtosecond to nanosecond time scales. ${ }^{[29]}$ Although the ultrafast femtosecond components are suggestive of "free" water molecules in bulk, ${ }^{[52]}$ the nanosecond components corresponding to slow solvation may arise from the motion of the probe molecule in and out of the cavity, fluctuations of the structural components comprising the CD cavity, and hindered reorientation of the highly restricted water molecules.

Zhang et al. used site-specific protein modification engineering to map the protein surface in terms of hydration dynamics and water-protein interactions. ${ }^{[35]}$ They used the intrinsic tryptophan residue as a molecular reporter to study the globular $\alpha$-helical protein apomyoglobin (apoMb) in its native $(\mathrm{N})$ and molten globule (MG) states. The relaxation dynamics, as interpreted in terms of the Stokes shift of the tryptophan emission, was found to be broadly bimodal, characterized by two time scales, $\tau_{1}$ and $\tau_{2}$. Although local variations in topology, hydrophobicity, and ambient charge densities at the different tryptophan sites resulted in different values for the native and molten globule forms, the initial water relaxation $\left(\tau_{1}\right)$ was found to occur within 10 ps $(\sim 0.7-8.2 \mathrm{ps})$; further, it was attributed to the local collective water network motions, thereby implying the rigidness of the $\mathrm{H}$-bond network in the protein hydration layer. In contrast, the slower relaxation time $\left(\tau_{2}\right)$ exhibited greater variation in magnitude, ranging from few tens of picoseconds to a couple of hundred picoseconds. The longer relaxation times were attributed to the interaction of interfacial water molecules in the inner hydration shell with the proteins. The extensive reorganization of the water network in accordance with the altered dipole moment of the probe is facilitated by local protein fluctuations and through the dynamic exchange between bound and bulk water. The coupling of such interactions results in protein-water collective motion.

Kwon et al. demonstrated that the surface modification of a coiled-coil protein through the inclusion of fluorinated residues can have a significant bearing on water-protein interactions and their consequent dynamics. ${ }^{[53]}$ Although fluorination often leads to enhanced hydrophobicity of the residues compared to that of their hydrogenated analogues, a larger size alone is not sufficient to explain the deceleration of the dynamics of biological water at such modified surfaces. Despite the low polarizability of the carbon-fluorine bond that thwarts its ability to participate in $\mathrm{H}$-bonding with water, dipolar interactions occur, and fluorinated side chains assert electrostatic attraction on the adjoining water molecules, thereby slowing down water mobility at the protein surface.

More recently, by conducting temperature-dependent solvation dynamics measurements on a model protein (DNA polymerase IV, Dpo4) and ten of its tryptophan (intrinsic fluorophore)-bearing mutants, Qin et al. have elucidated the critical role played by protein hydration shell fluctuations in dictating the protein side chain dynamics in picosecond timescales. ${ }^{[46]}$ They performed this study on such mutants to reveal the dynamics and energetics of biological water in four distinct domains of their model protein. The time-dependent solvent correlation functions of such water molecules were tracked in terms of three time components that varied from hundreds of femtoseconds to a few tens of picoseconds.

Concurrently, the side chain relaxation behavior was monitored in terms of four anisotropy decay components of the tryptophan residue. Of these four, only two components, attributed to the local wobbling relaxations, were correlated with the components derived from solvation dynamics. Several new insights emerged from their investigations once they related the temperature dependence of these components to Arrhenius-type behavior. First, the energy barriers and prefactors suggest that the buried tryptophan primarily forms a strong high-energy-barrier hydration network near the protein. Second, the similar magnitudes of the activation barriers for the hydration water and tryptophan indicate the common origin of their corresponding relaxation behaviors. Third, the distribution of barrier energies and pre-factors for broadly two different types of sites, namely buried and exposed, suggest that while the exposed dynamics conform to earlier reports, the motions in buried sites are typically faster than those hinted in previous studies. Fourth, hydration-associated pre-factors are always larger than those corresponding to tryptophan-driven relaxation. Fifth, certain domains can exhibit hydration features that comprise both ultrafast and slow dynamics, characteristic of deep-seated tryptophan residues. Taken together, these observations suggest the significant length scales of the extended biological water network containing the protein surface and reveal its collective dynamical motion, a key aspect that affects its function.

However, it is substantially challenging to interpret the activation energies of biological water by analyzing solvent correlation functions. For instance, the energy needed to populate a reactive configuration along a reaction coordinate depends not only on the temperature, but also on other factors such as the protein conformation. Park et al. have recently investigated the energetics of biological water by probing the rate of ESPT at protein surfaces. ${ }^{[54]}$ The phenomenon of proton transfer is strongly dependent on the local $\mathrm{H}$-bonding network; accordingly, in the vicinity of bio-macromolecules, it can vary significantly from that in the bulk..$^{[57-64]}$ 
Given the complexities associated with the interpretation of activation energies that depend on temperature and macromolecular conformation, ESPT was proposed as an alternate isothermal probing phenomenon that can be exploited to obtain important experimental insights regarding the characteristics of biological water around proteins. Park et al. employed 7-azatryptophan (AW), a non-canonical amino acid, whose prototropic chromophore (7-azaindole, 7AI) has been the subject of several photophysical investigations. ${ }^{[65,66]}$ The key to understanding the role of 7AI lies in the fact that its ESPT is catalyzed by a water molecule and involves the formation of a

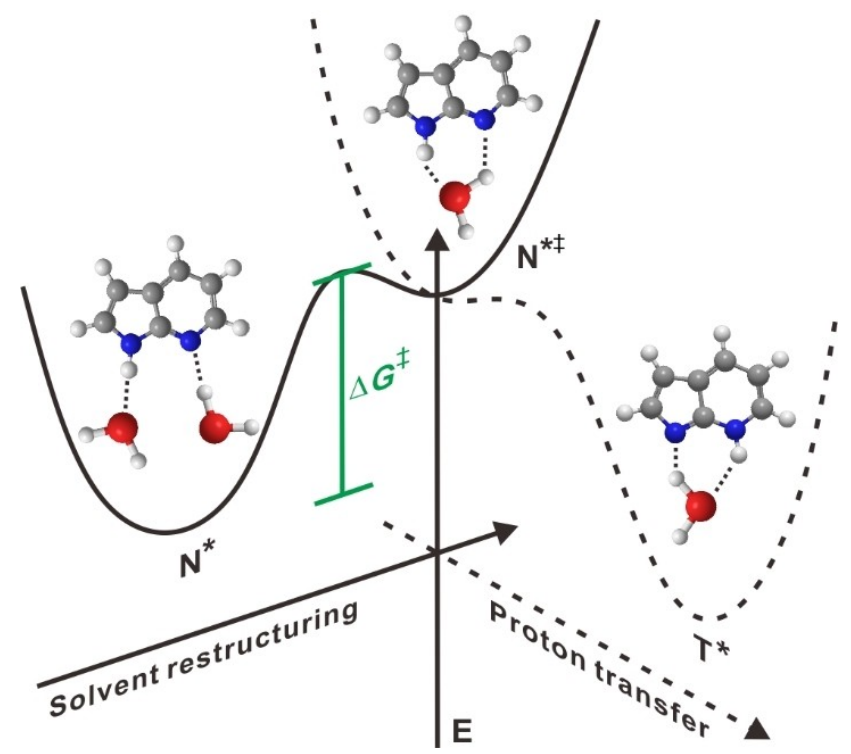

Figure 3. Schematic energy diagram of the excited-state proton-transfer model of 7-azaindole. Modified from Ref. [62], copyright 2005, with permission from the American Chemical Society. transient cyclical H-bonded 1:1 complex with the parent photoexcited normal form (Figure 3). ${ }^{[62-64]}$ The stability of this transient intermediate is dictated by the ease, with which it can associate with an ambient water molecule, which in turn is affected by the dynamic restructuring of the $\mathrm{H}$-bond network.

Spoel's method was applied to the result of MD simulations for the estimation of the differences between the free energies of $\mathrm{H}$-bonds in biological and bulk water. Accordingly, the kinetics of $\mathrm{H}$-bond breakage and re-formation were treated in terms of a reactive-flux correlation function. The $\mathrm{H}$-bond lifetime was calculated using the inverse of the forward rate constant, and the Gibbs energy of activation was estimated under the assumption that $\mathrm{H}$-bond breakage may be regarded as an Eyring process.

The analyses of the experimentally measured proton-transfer rates revealed that the $\mathrm{H}$-bond free energy of biological water in the vicinity of the AW site was $0.4 \mathrm{kcal} \mathrm{mol}^{-1}$ higher than that of bulk water (Figure 4). They attributed this difference to the entropic costs incurred in sustaining a highly ordered $\mathrm{H}$-bond network around the charged and hydrophilic surface of the protein. Both ion size and the topology of the biological surface help govern the energetics of such $\mathrm{H}$-bonds. It may be noted that the use of the ESPT model to estimate the $\mathrm{H}$-bond energetics is effective for probing water at protein surfaces, where the rate of ESPT is modulated only by the characteristic $\mathrm{H}$-bond strengths of the biological water molecules. Such a method may not be feasible for probing water buried within protein cavities.
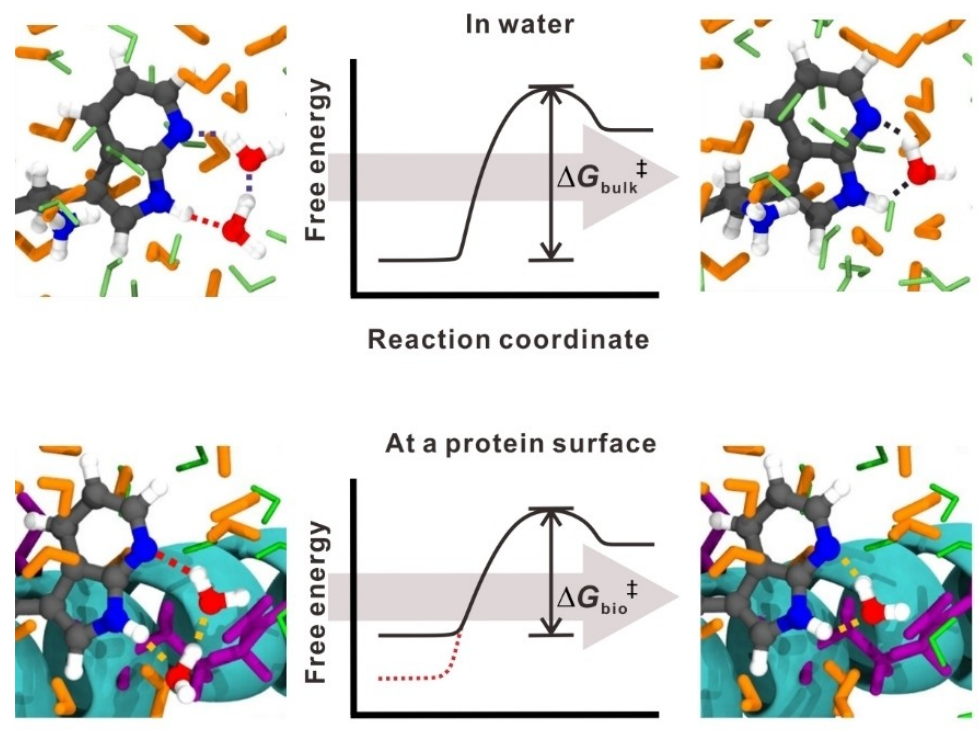

Reaction coordinate

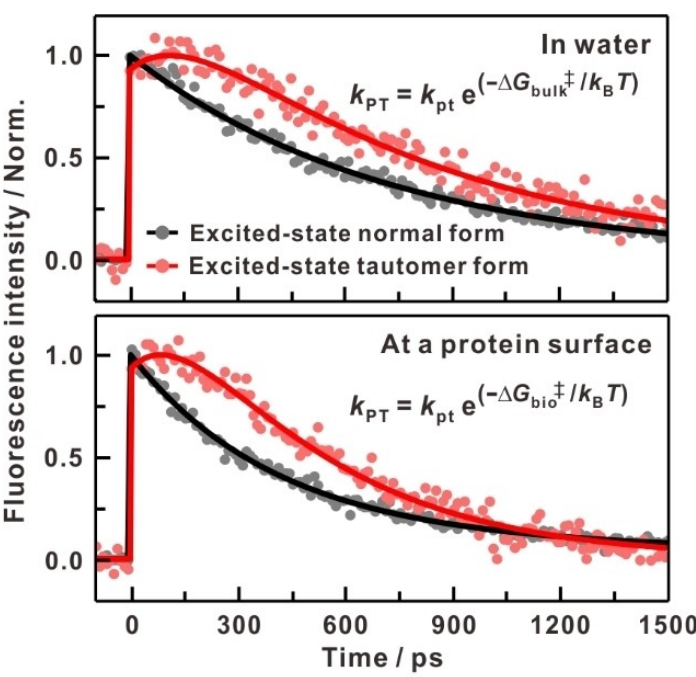

$\Delta G$ for H-bond breakage of biological water

$$
\simeq \Delta G_{\mathrm{bio}^{\ddagger}} \neq-\Delta \boldsymbol{G}_{\mathrm{bulk}}{ }^{\ddagger}
$$

Figure 4. Schematic comparison of the H-bonding energetics of water in bulk and in the vicinity of the model protein surface probed by the proton-transfer reaction to measure the $\mathrm{H}$-bond free-energy difference $(\Delta \Delta \mathrm{G})$ between bulk water and biological water. Reproduced from Ref. [54], copyright 2020, with permission from Wiley-VCH Verlag $\mathrm{GmbH} \& \mathrm{Co}$. 


\subsection{Computational Studies}

A substantial number of computational studies have been conducted on the high molecular-length-scale heterogeneity of biological water with regard to the interpretation of protein hydration. Molecular dynamics (MD) simulations are often used for correlating the translational and rotational diffusion coefficients of water molecules in the hydration layer with their residence time on protein sites. ${ }^{[67]}$ The trajectory analysis of tagged molecules suggests the coexistence of the "bound" and "free" water states, which is characterized by rapid dynamic exchange between the two. Such equilibrium can be understood using a theoretical framework that treats the exchange in terms of a double well potential near the surface (Figure 1a). The sluggishness of biological water is thus attributed not only to the fact that biological water is constrained within the $\mathrm{H}$ bonded hydration layer, but also to its ability to revert back to the bound state once it is free. The mechanism and rate of $\mathrm{H}$ bond breakage within the protein hydration layers are often studied using time correlation function techniques. The bondbreaking behavior appreciably differs from that of bulk water owing to the constrained nature of the $\mathrm{H}$-bond network. In particular, the reorientation processes are associated with $\mathrm{H}$ bond breaking/switching events. Besides reorientation, the $\mathrm{H}$ bond lifetime dynamics also provides a tool to gauge the unique dynamics of biological water. Although the time constant for the decay exhibited by the $\mathrm{H}$-bond lifetime correlation function increases from 1.8 to $2.6 \mathrm{ps}$ in hydration layers, there exists a distribution in the $\mathrm{H}$-bond stability depending on whether the adjoining biomolecule surface is hydrophilic or hydrophobic. The residence time may also depend on the location of water along a protein; particularly, water near backbone atoms show lower average characteristic correlation decay times than that at side-chain atoms.

Using several proteins that were chosen for the diversity of their structure, function, and helix-sheet ratio, Mukherjee et al. showed how the broad distribution of residence times and rotation relaxation times of water molecules within the protein hydration layer is responsible for the non-exponential nature of the dielectric response often observed for biological water. ${ }^{[68-71]}$ Water molecules bound to peptide backbones around hydrophobic residues can be more mobile than bulk water molecules. In contrast, those bound to charged amino acid groups show substantial retardation of the associated dynamics. Interestingly, the nuances of such a variegated scenario are lost when only the average time constants are considered. Averaging yields values that are not orders of magnitude, but merely 2 to 3 times slower than bulk water. Such estimates of time constants erroneously suggest the dynamics of hydration layer water as being bulk-like. These values appear consistent with the estimates derived from NMR measurements, a technique that, unlike time-dependent fluorescence Stokes' shift (TDFSS), provides only average values involving both surface and bulk water molecules resulting in biasing toward faster relaxation. In other studies, Mondal et al. focused on tryptophan solvation in different proteins and introduced mutations in the charge distribution around the intrinsic probe. ${ }^{[69]}$ Dynamic coupling between amino acid side chains and water molecules was probed and amino acid side chain motion was found to be essential for solvation components with time constants of $100 \mathrm{ps}$ or longer. The residual slow component in the range of a few tens of picosecond was attributed to slow water molecules within the protein hydration layer. This assessment is consistent with TDFSS studies in which such a component is found to contribute less than $20 \%$ of the total decay, irrespective of the protein's nature. ${ }^{[26-28]}$ In their measurements using coiled-coil proteins, Park et al. observed solvation time components with amplitudes on the order of $20 \%{ }^{[54]} \mathrm{A}$ component of approximately $40 \mathrm{ps}$ was also observed through dielectric relaxation measurements of aqueous protein solutions to probe the hydration shell in the neighborhood of protein surfaces. ${ }^{[41]}$ This component for biological water was absent in NMR measurements, where the contribution of the slow water molecules to the overall dynamics is swamped by the faster bulk water molecules.

$\mathrm{Li}$ et al. analyzed water and protein dynamics in apoMb solutions using MD simulations to understand various contributions to the experimentally observed bi-modal relaxation behavior, particularly the slower relaxation component. ${ }^{[72]}$ They selectively froze the protein motion in their simulations to isolate and estimate the contribution of water molecules in the protein hydration layer. They also constrained the water motions to determine the influence of solvent dynamics on protein fluctuations. They showed that the initial relaxation in 5 ps following the photoexcitation of the tryptophan residue is characterized by local motions that include the translation and reorientation of water molecules within the hydration layer (Figure 5). The slow component of the Stokes shift extending over tens of picoseconds ( $90 \mathrm{ps)}$ can only be recovered in the presence of strongly coupled protein-water fluctuations and is eliminated when either protein or water is constrained. Li et al. also compared their results and conclusions with those of a previous simulation study on monellin protein, according to which the sluggish component of the Stokes shift is caused by the protein motion alone, ${ }^{[73]}$ hence, they argued that the sluggishness of biological water is owing to the flexibility of the protein (and water), even when the contribution of a slow protein component to the Stokes shift was not evident.

MD simulations conducted by Park et al. ${ }^{[54]}$ on AW bearing engineered coiled-coil proteins revealed that parameters such as the $\mathrm{H}$-bond lifetimes and activation energy needed to break $\mathrm{H}$-bonds between water molecules at different hydration layers of the probing moiety can be effectively employed to estimate the $\mathrm{H}$-bond energies of biological water. They estimated the $\mathrm{H}$ bonding free energy difference between bulk and biological water molecules to be $0.18,0.29$, and $0.33 \mathrm{kcal} \mathrm{mol}^{-1}$ along each hydration shell. This result implied that the biological water molecules tend to be energetically more fragile than bulk water molecules, which is a consequence of an intricate interplay between various factors such as the local topology of the biomolecular surface, the nature and size of the charged residues in the immediate vicinity, and the associated counter-ions. 


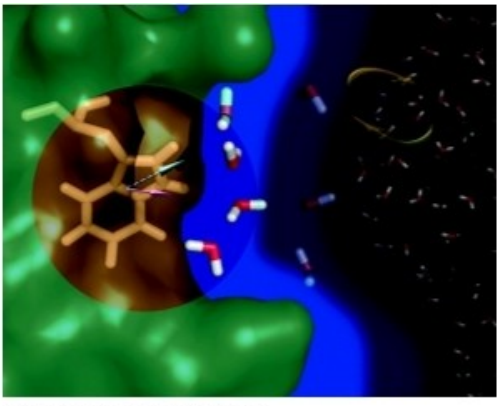

Hydration dynamics

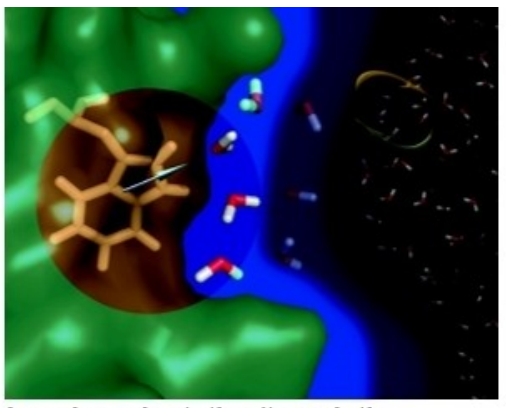

Local reorientation/translation

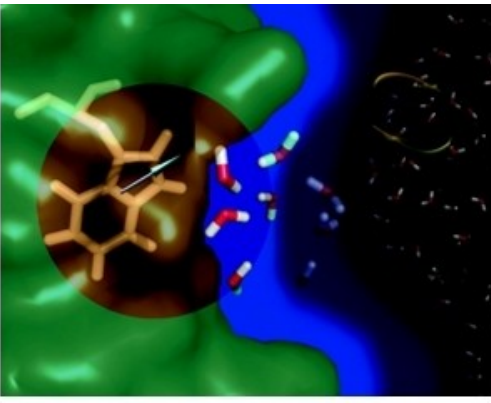

Coupled water-protein motion

$t=0 \mathrm{ps}$

$t=5 \mathrm{ps}$

$t=87 \mathrm{ps}$

Figure 5. MD simulation trajectory for protein surface hydration at a tryptophan site with two adjacent layers of hydration. Modified from Ref. [72], copyright 2007, with permission from the American Chemical Society.

\section{Summary and Outlook}

Although "biological water" is characterized by physical chemists in terms of $\mathrm{H}$-bond strengths, density, and orientation, among others, as reflected by its dynamics and energetics, the insights gleaned from such characterization need not be irrelevant to the wider biochemistry community. For example, the average distance of $1 \mathrm{~nm}$ between macromolecules within the cytoplasm underlies the fact that water within cells are always under the influence of several macromolecules and therefore should not be expected to be "bulk-like". ${ }^{[71]}$ The macromolecular crowding within cells can lead to enhanced viscosity and lower diffusivity for intracellular water. ${ }^{[4]}$ Depending on the features of the intracellular environment, the abundance and strength of the $\mathrm{H}$-bond network are expected to vary, leading to the emergence of aqueous phases with slightly different hydration characteristics. Such macroscopic restructuring of the $\mathrm{H}$-bond network should, in turn, dictate the reactivity and metabolic capabilities of biological entities, leading to alterations in cellular activity. The low dielectric constant of the protein hydration layer may facilitate interactions between water molecules in the layer as well as between the water molecules and substrates or ligands. The energetic distribution across the different types of biological water indicates a larger specific heat for the hydration layer compared to bulk, and this in turn may help stabilize protein structures by minimizing temperature fluctuations in the protein-associated water. ${ }^{[68]}$

All ultrafast physicochemical phenomena investigated to elucidate the dynamics and energetics of biological water, such as solvation dynamics, excited-state proton transfer, and depolarization dynamics, depend on different aspects of the hydration environment surrounding a fluorophore, including the local polarity, H-bond network, and micro-viscosity of the medium. The most important aspect that should be considered, while designing experiments to understand the dynamics of biological water, is the need to minimize any perturbation of the biological structures caused by the incorporation of the probe into the system. Site-specific mutations of proteins with highly environment-sensitive probes offer the prospect of examining various aspects of biological water, including the various topologies observed among different domains within a given protein or those observed between hydrophilic and hydrophobic moieties of proteins that govern their secondary structures and aid their stability and functionality. The prospect of using non-canonical amino acids through protein engineering provides potential opportunities to expand understanding of the dynamics of water-protein interactions, which can be utilized to manipulate the functional characteristics of proteins, such as binding and molecular recognition by tailoring protein surfaces (and cavities).

\section{Acknowledgements}

This work was supported by the Institute for Basic Science (IBSR020-D1), Korea, and the National Research Foundation of Korea (NRF) funded by the Ministry of Science, ICT, and Future Planning (MSIP) (2014R1A1A1008289).

\section{Conflict of Interest}

The authors declare no conflict of interest.

Keywords: biological water - excited-state proton transfer $\mathrm{H}$-bond dynamics · protein hydration · solvation dynamics

[1] B. Bagchi, Water in Biological and Chemical Processes, Cambridge University Press, New York, 2013.

[2] P. Ball, ChemPhysChem 2008, 9, 2677-2685

[3] P. Ball, Proc. Natl. Acad. Sci. USA 2017, 114, 13327-13335.

[4] M. Chaplin, Nat. Rev. Mol. Cell Biol. 2006, 7, 861-866.

[5] P. Jungwirth, J. Phys. Chem. Lett. 2015, 6, 2449-2451.

[6] Y. Levy, J. N. Onuchic, Annu. Rev. Biophys. Biomol. Struct. 2006, 35, 389415.

[7] P. L. Privalov, C. Crane-Robinson, Eur. Biophys. J. 2017, 46, 203-224.

[8] E. A. Vogler, Adv. Colloid Interface Sci. 1998, 74, 69-117.

[9] P. M. Wiggins, Microbiol. Rev. 1990, 54, 432-449. 
[10] A. Adhikari, S. Re, W. Nishima, M. Ahmed, S. Nihonyanagi, J. B. Klauda, Y. Sugita, T. Tahara, J. Phys. Chem. C 2016, 120, 23692-23697.

[11] Y. Fujiyoshi, K. Mitsuoka, B. L. de Groot, A. Philippsen, H. Grubmüller, P. Agre, A. Engel, Curr. Opin. Struct. Biol. 2002, 12, 509-515.

[12] H. Shweta, N. Pal, M. K. Singh, S. D. Verma, S. Sen, in C. Geddes (eds), Reviews in Fluorescence 2017, Reviews in Fluorescence, Springer, Cham, 2018.

[13] J. K. Kim, C. Lee, S. W. Lim, J. T. Andring, A. Adhikari, R. McKenna, C. U. Kim, IUCrJ. 2020, 7, 985-994.

[14] J. K. Kim, C. Lee, S. W. Lim, A. Adhikari, J. T. Andring, R. McKenna, C.-M. Ghim, C. U. Kim, Nat. Commun. 2020, 11, 4557.

[15] N. Nandi, B. Bagchi, J. Phys. Chem. B 1997, 101, 10954-10961.

[16] R. Biswas, B. Bagchi, J. Phys. Condens. Matter 2018, 30, 013001.

[17] Y. Maréchal, The Hydrogen Bond and the Water Molecule, Elsevier, Amsterdam, 2007.

[18] G. Gilli, P. Gilli, The Nature of the Hydrogen Bond, Oxford University Press Inc., New York, 2009.

[19] K. Bhattacharyya, Chem. Commun. 2008, 2848-2857.

[20] D. Laage, T. Elsaesser, J. T. Hynes, Chem. Rev. 2017, 117, 10694-10725.

[21] I. Brovchenko, A. Oleinikova, ChemPhysChem 2008, 9, 2695-2702.

[22] T. Tsuruta, J. Biomater. Sci. Polym. Ed. 2010, 21, 1831-1848.

[23] T. M. Raschke, Curr. Opin. Struct. Biol. 2006, 16, 152-159.

[24] G. Schirò, M. Weik, J. Phys. Condens. Matter 2019, 31, 463002.

[25] H. E. Stanley, S. V. Buldyrev, G. Franzese, P. Kumar, F. Mallamace, M. G. Mazza, K. Stokely, L. Xu, J. Phys. Condens. Matter 2010, 22, 284101.

[26] D. Zhong, S. K. Pal, A. H. Zewail, Chem. Phys. Lett. 2011, 503, 1-11.

[27] S. K. Pal, J. Peon, B. Bagchi, A. H. Zewail, J. Phys. Chem. B 2002, 106, 12376-12395

[28] S. K. Pal, A. H. Zewail, Chem. Rev. 2004, 104, 2099-2123.

[29] Š. Vajda, R. Jimenez, S. J. Rosenthal, V. Fidler, G. R. Fleming, E. W. Castner, Jr., J. Chem. Soc. Faraday Trans. 1995, 91, 867-873.

[30] G. A. Jeffrey, J. Mol. Struct. 1994, 322, 21-25.

[31] S. Pal, S. Balasubramanian, B. Bagchi, J. Phys. Chem. B 2003, 107, 5194 5202.

[32] A. Adhikari, J.-H. Park, H.-W. Nho, O.-H. Kwon, J. Mol. Liq. 2020, 320 114346.

[33] S. M. Bhattacharyya, Z.-G. Wang, A. H. Zewail, J. Phys. Chem. B 2003, 107, 13218-13228.

[34] M. Levitt, R. Sharon, Proc. Natl. Acad. Sci. USA 1988, 85, 7557-7561.

[35] L. Zhang, Y. Yang, Y.-T. Kao, L. Wang, D. Zhong, J. Am. Chem. Soc. 2009, $131,10677-10691$

[36] A. Ben-Naim, J. Phys. Chem. 1991, 95, 1437-1444.

[37] E. Tajkhorshid, P. Nollert, M. Ø. Jensen, L. J. W. Miercke, J. O'Connell, R. M. Stroud, K. Schulten, Science 2002, 296, 525-530.

[38] K. Brejc, T. K. Sixma, P. A. Kitts, S. R. Kain, R. Y. Tsien, M. Ormö, S. J. Remington, Proc. Natl. Acad. Sci. USA 1997, 94, 2306-2311.

[39] A. Shinobu, N. Agmon, J. Phys. Chem. B 2015, 119, 3464-3478.

[40] Y. Erez, D. Huppert, J. Phys. Chem. A 2010, 114, 8075-8082.

[41] R. Pethig, Annu. Rev. Phys. Chem. 1992, 43, 177-205.

[42] D. W. Pierce, S. G. Boxer, J. Phys. Chem. 1992, 96, 5560-5566.

[43] B. Halle, M. Davidovic, Proc. Natl. Acad. Sci. USA 2003, 100, 12135-12140.

[44] V. C. Nibali, M. Havenith, J. Am. Chem. Soc. 2014, 136, 12800-12807.

[45] R. K. Mitra, S. S. Sinha, S. K. Pal, J. Phys. Chem. B 2007, 111, 7577-7583.

[46] Y. Qin, L. Wang, D. Zhong, Proc. Natl. Acad. Sci. USA 2016, 113, 8424 8429.
[47] P. Sen, S. Mukherjee, A. Halder, K. Bhattacharyya, Chem. Phys. Lett. 2004, 385, 357-361.

[48] M. Kumbhakar, T. Goel, T. Mukherjee, H. Pal, J. Phys. Chem. B 2004, 108, 19246-19254.

[49] P. Abbyad, X. Shi, W. Childs, T. B. McAnaney, B. E. Cohen, S. G. Boxer, J. Phys. Chem. B 2007, 111, 8269-8276.

[50] S. K. Pal, D. Mandal, D. Sukul, S. Sen, K. Bhattacharyya, J. Phys. Chem. B 2001, 105, 1438-1441.

[51] X. J. Jordanides, M. J. Lang, X. Song, G. R. Fleming, J. Phys. Chem. B 1999, 103, 7995-8005.

[52] R. Jimenez, G. R. Fleming, P. V. Kumar, M. Maroncelli, Nature 1994, 369, 471-473.

[53] O.-H. Kwon, T. H. Yoo, C. M. Othon, J. A. Van Deventer, D. A. Tirrell, A. H. Zewail, Proc. Natl. Acad. Sci. USA 2010, 107, 17101-17106.

[54] W.-W. Park, K. M. Lee, B. S. Lee, Y. J. Kim, S. H. Joo, S. K. Kwak, T. H. Yoo, O.-H. Kwon, Angew. Chem. Int. Ed. 2020, 59, 7089-7096.

[55] K. Wendler, J. Thar, S. Zahn, B. Kirchner, J. Phys. Chem. A 2010, 114, 9529-9536.

[56] Y. Harano, Entropy 2012, 14, 1443-1468.

[57] T. H. Nguyen, C. Zhang, E. Weichselbaum, D. G. Knyazev, P. Pohl, P. Carloni, PLoS One 2018, 13, 0193454.

[58] J.-Y. Shen, W.-C. Chao, C. Liu, H.-A. Pan, H.-C. Yang, C.-L. Chen, Y.-K. Lan, L.-J. Lin, J.-S. Wang, J.-F. Lu, S. C.-W. Chou, K.-C. Tang, P.-T. Chou, Nat. Commun. 2013, 4, 2611.

[59] E. Weichselbaum, M. Österbauer, D. G. Knyazev, O. V. Batishchev, S. A Akimov, T. H. Nguyen, C. Zhang, G. Knör, N. Agmon, P. Carloni, P. Pohl, Sci. Rep. 2017, 7, 4553.

[60] J. W. Mazzuca, C. P. Schultz, J. Phys. Chem. A 2017, 121, 819-826.

[61] M. Brändén, T. Sandén, P. Brzezinski, J. Widengren, Proc. Natl. Acad. Sci. USA 2006, 103, 19766-19770.

[62] O.-H. Kwon, D.-J. Jang, J. Phys. Chem. B 2005, 109, 20479-20484.

[63] C. F. Chapman, M. Maroncelli, J. Phys. Chem. 1992, 96, 8430-8441.

[64] S. Mente, M. Maroncelli, J. Phys. Chem. A 1998, 102, 3860-3876.

[65] K. L. Rich, M. Négrerie, J. Li, S. Elliott, R. W. Thornburg, J. W. Petrich, Photochem. Photobiol. 1993, 58, 28-30.

[66] V. De Filippis, S. De Boni, E. De Dea, D. Dalzoppo, C. Grandi, A. Fontana, Protein Sci. 2004, 13, 1489-1502.

[67] B. Jana, S. Pal, B. Bagchi, J. Phys. Chem. B 2008, 112, 9112-9117.

[68] S. Mukherjee, S. Mondal, B. Bagchi, J. Chem. Phys. 2017, 147, 024901.

[69] S. Mondal, S. Mukherjee, B. Bagchi, J. Chem. Phys. 2017, 147, 154901.

[70] S. Mukherjee, S. Mondal, B. Bagchi, Phys. Rev. Lett. 2019, 122, 058101.

[71] S. Mondal, S. Mukherjee, B. Bagchi, J. Phys. Chem. Lett. 2017, 8, 48784882.

[72] T. Li, A. A. Hassanali, Y.-T. Kao, D. Zhong, S. J. Singer, J. Am. Chem. Soc. 2007, 129, 3376-3382.

[73] L. Nilsson, B. Halle, Proc. Natl. Acad. Sci. USA 2005, 102, 13867-13872.

Manuscript received: October 20, 2020

Revised manuscript received: November 26, 2020

Accepted manuscript online: November 29, 2020 